Electron Emission from Slightly Oxidized Depleted Uranium Generated by its Own Radioactivity Measured by Electron Spectroscopy, and Electron-Induced Dissociation and Ionization of Hydrogen Near its Surface.

W. J. Siekhaus, A. J. Nelson

October 27, 2011 
This document was prepared as an account of work sponsored by an agency of the United States government. Neither the United States government nor Lawrence Livermore National Security, LLC, nor any of their employees makes any warranty, expressed or implied, or assumes any legal liability or responsibility for the accuracy, completeness, or usefulness of any information, apparatus, product, or process disclosed, or represents that its use would not infringe privately owned rights. Reference herein to any specific commercial product, process, or service by trade name, trademark, manufacturer, or otherwise does not necessarily constitute or imply its endorsement, recommendation, or favoring by the United States government or Lawrence Livermore National Security, LLC. The views and opinions of authors expressed herein do not necessarily state or reflect those of the United States government or Lawrence Livermore National Security, LLC, and shall not be used for advertising or product endorsement purposes.

This work performed under the auspices of the U.S. Department of Energy by Lawrence Livermore National Laboratory under Contract DE-AC52-07NA27344. 


\title{
Electron emission from slightly oxidized depleted Uranium generated by its own radioactivity measured by electron spectroscopy, and electron- induced dissociation and ionization of hydrogen near its surface.
}

\author{
Wigbert Siekhaus, Art J. Nelson \\ Lawrence Livermore National Laboratory
}

\begin{abstract}
Energy dependent electron emission (counts per second) between zero and $1.4 \mathrm{keV}$ generated by the natural reactivity of uranium was measured by an electrostatic spectrometer with known acceptance angle and acceptance area. The electron intensity decreases continuously with energy, but at different rates in different energy regimes, suggesting that a variety of processes may be involved in producing the observed electron emission. The spectrum was converted to energy dependent electron flux $\left(\mathrm{e}-/ \mathrm{cm}^{2} \mathrm{~s}\right)$ using the assumption that the emission has a cosine angular distribution. The flux decreased rapidly from $\sim 10^{6} / \mathrm{cm}^{2}$ s to $\sim 10^{5} / \mathrm{cm}^{2} \mathrm{~s}$ in the energy range from zero to $200 \mathrm{eV}$, and then more slowly from $\sim 10^{5} / \mathrm{cm}^{2}$ s to $\sim 3^{*} 10^{4} / \mathrm{cm}^{2} \mathrm{~s}$ in the range from 200 to $1400 \mathrm{eV}$. The energy dependent electron mean free path in gases together with literature cross sections for electron induced reactions were used to determine the number of ionization and dissociation reactions per $\mathrm{cm}^{2} \mathrm{~s}$ within the inelastic mean free path of electrons, and found to be about $1.3^{*} 10^{8} / \mathrm{cm}^{2} \mathrm{~s}$ and $1.5^{*} 10^{7} / \mathrm{cm}^{2} \mathrm{~s}$, respectively, for hydrogen. An estimate of the number of ionization and dissociation reactions occurring within the total range, rather than the mean free path of electrons in gases resulted in $6.2 * 10^{9} / \mathrm{cm}^{2} \mathrm{~s}$ and $1.3^{*} 10^{9} / \mathrm{cm}^{2} \mathrm{~s}$, respectively.

The total energy flux carried by electrons from the surface is suspiciously close to the total possible energy generated by one gram of uranium. A likely source of error is the assumption that the electron emission has a cosine distribution. Angular distribution measurements of the electron emission would check that assumption, and actual measurement of the total current emanating from the surface are needed to confirm the value of the current calculated in section II.

These results must therefore be used with caution - until they are confirmed by other measurements.
\end{abstract}

This work performed under the auspices of the U.S. Department of Energy by Lawrence Livermore National Laboratory under Contract DE-AC52-07NA27344 


\section{I) Experimental Results.}

Electron emission from an oxidized surface of depleted Uranium into vacuum was measured using the analyzer ${ }^{1}$ of an X-ray Photoelectron Spectroscopy System (XPS system) while its X-ray source was turned off. The spectrometer's input axis is at an angle $\theta=45$ to the sample surface, and the acceptance area is $300 \mu \mathrm{m} * 800 \mu \mathrm{m}$. The spectrometer has an acceptance angle $\Phi$ of $\pm 20^{\circ}$. The ionization gauge was "on" during the run and during a background counting run with a non-radioactive sample in front of the analyzer and the Uranium sample on the sample holder, but removed from the acceptance area of the of the spectrometer. The results are shown in figure 1.

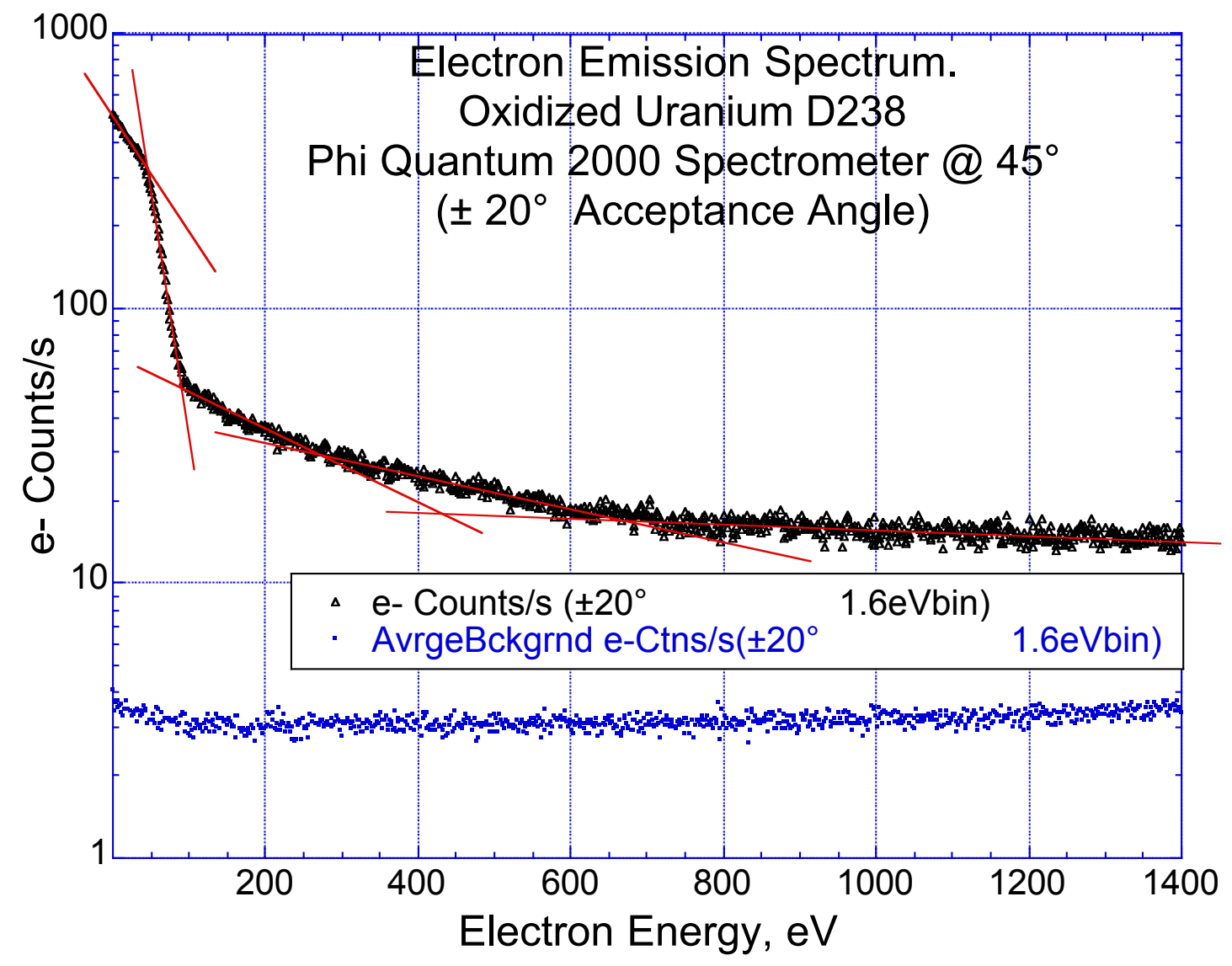

Figure 1. Electron count rate (counts per second) from an oxidized Uranium surface. Signal with background counts are shown as black triangles, background counts as blue squares.

The spectrum appears to consist of 5 segments with different exponential slopes, suggesting that different generating processes are involved. Electrons at these energies have a mean free path of only a few mono-layers (see figure 5). Hence they originate from the Uranium-oxide layers and their generating processes may involve

1 Physical Electronics PHI Quantum 2000, Scanning ESCA System 
electronic structure characteristics of Uranium Oxide. The total number of counted electrons $=\int$ Counts(E)dE is 30276, and the total emitted energy of the collected electrons is $\left(=\int \mathrm{E}^{*} \operatorname{Counts}(\mathrm{E}) \mathrm{dE}\right)$ is $9.2^{*} 10^{\wedge} 6 \mathrm{eV} / \mathrm{s}, 1.5^{*} 10^{\wedge}-12$ Watt.

\section{II ) Conversion of experimental results (counts/s) to electron flux $\left(e-/ \mathrm{cm}^{2} \mathbf{s}\right)$}

The data of figure 1 (counts/s) must be converted to "electrons emitted/ $\mathrm{cm}^{2} \mathrm{~s}$ " to calculate electron-induced chemistry, as illustrated in figure 3 below.

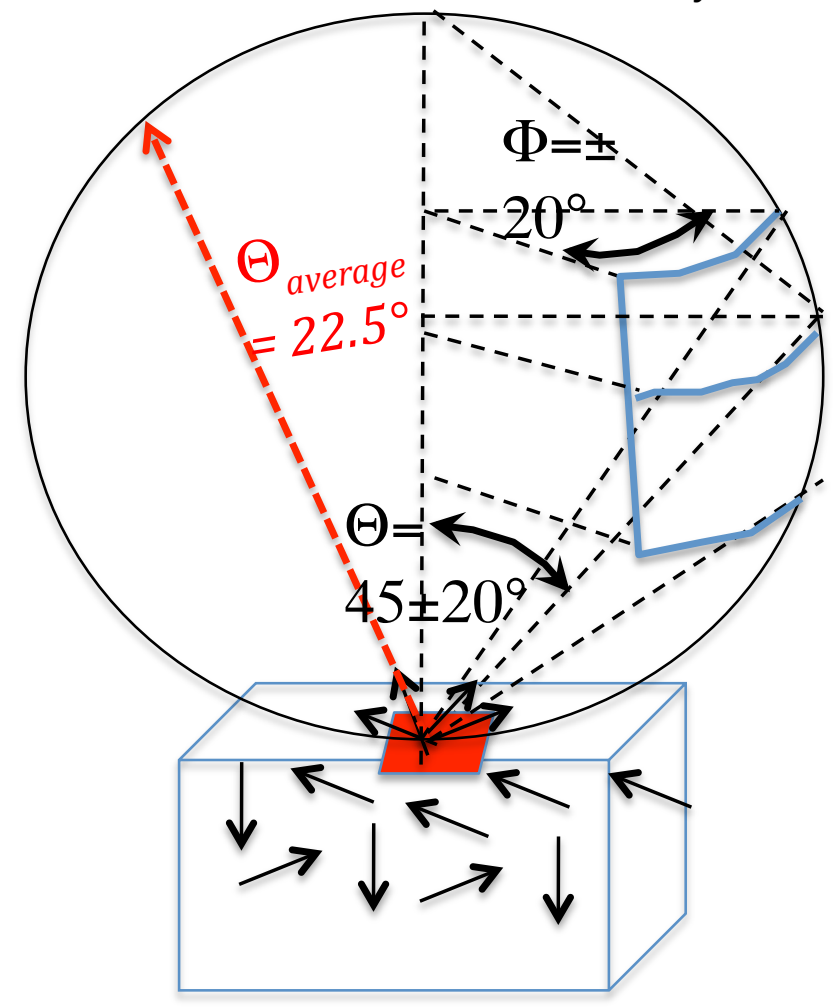

Flux from source area: $=\mathrm{N}_{0} / \mathrm{cm}^{2} \mathrm{~s}$, Flux in cone of angle $\Theta=45 \pm 20^{\circ}, \Phi= \pm 20^{\circ}$ :

$\int_{\Theta=45-20^{\circ}}^{\Theta=45+20^{\circ}} \int_{\Phi=-20^{\circ}}^{\Phi=+20^{\circ}} N_{0} \cos \vartheta \sin \vartheta d \Phi d \vartheta=.222 \pi N_{0} \int_{\Theta=45-20^{\circ}}^{\Theta=45+20^{\circ}} \cos \vartheta \sin \vartheta d \vartheta=.2222 \pi N_{0} * .321394=.071421 \pi N_{0}$

The integral over all angles $(\Theta=0->\pi / 2$ and all $\Phi)$ equals $=2 \pi N_{0} * .5=\pi N_{0}$

The angle $\Theta_{\text {average }}$ into which 'on average' electrons are emitted

$\Theta_{\text {average }}=\frac{\int_{\vartheta=0}^{\pi / 2} \int_{\varphi=0}^{2 \pi} N_{0} \vartheta \cos \vartheta \sin \vartheta d \Phi d \vartheta}{\int_{\vartheta=0}^{\pi / 2} \int_{\varphi=0}^{2 \pi} N_{0} \cos \vartheta \sin \vartheta d \Phi d \vartheta}=.392699->22.5^{\circ}$

Figure 2. The electron emission from a substrate in which the electron flux inside the substrate is $\mathrm{N}_{0}$ electrons $/ \mathrm{cm}^{2} \mathrm{~s}$ for a $\mathrm{cm}^{2}$ area of ANY orientation. With that assumption the flux from the surface has a "cosine distribution" with an 'average' emission angle of $22.5^{\circ}$, and the fraction of the total flux sampled by the spectrometer with an acceptance angle of $\pm 20^{\circ}$ is .071421 of $\mathrm{N}_{0}$. 
The analysis in figure 2 assumes that electrons inside the sample travel in all directions with equal probability. The spectrometer has an entrance aperture of $4 \mathrm{mmx} 10 \mathrm{~mm}$. Its input lens demagnifies the size of the aperture by approximately $12 \mathrm{x}$, resulting in an acceptance area of approximately $300 \mathrm{x} 800 \mu \mathrm{m}$ when the sample is helt at the focal point of the input lens ${ }^{2}$. The spectrometer is positioned at an angle $45^{\circ}$ to the surface. Figure 2 shows that only .071421 of the total flux emanating from the surface area it "sees" of $\left(.3 \mathrm{mmx} .8 \mathrm{~mm} / \sin 45^{\circ}\right)=3.38^{-3} \mathrm{~cm}^{2}$ is collected by the spectrometer. Hence the counts per second of figure 1 must be divided by $\left(.071421 * 3.38^{-3}\right)$ to yield electrons $/ \mathrm{cm}^{2} \mathrm{~s}$. The results of this division are plotted in figure 3.

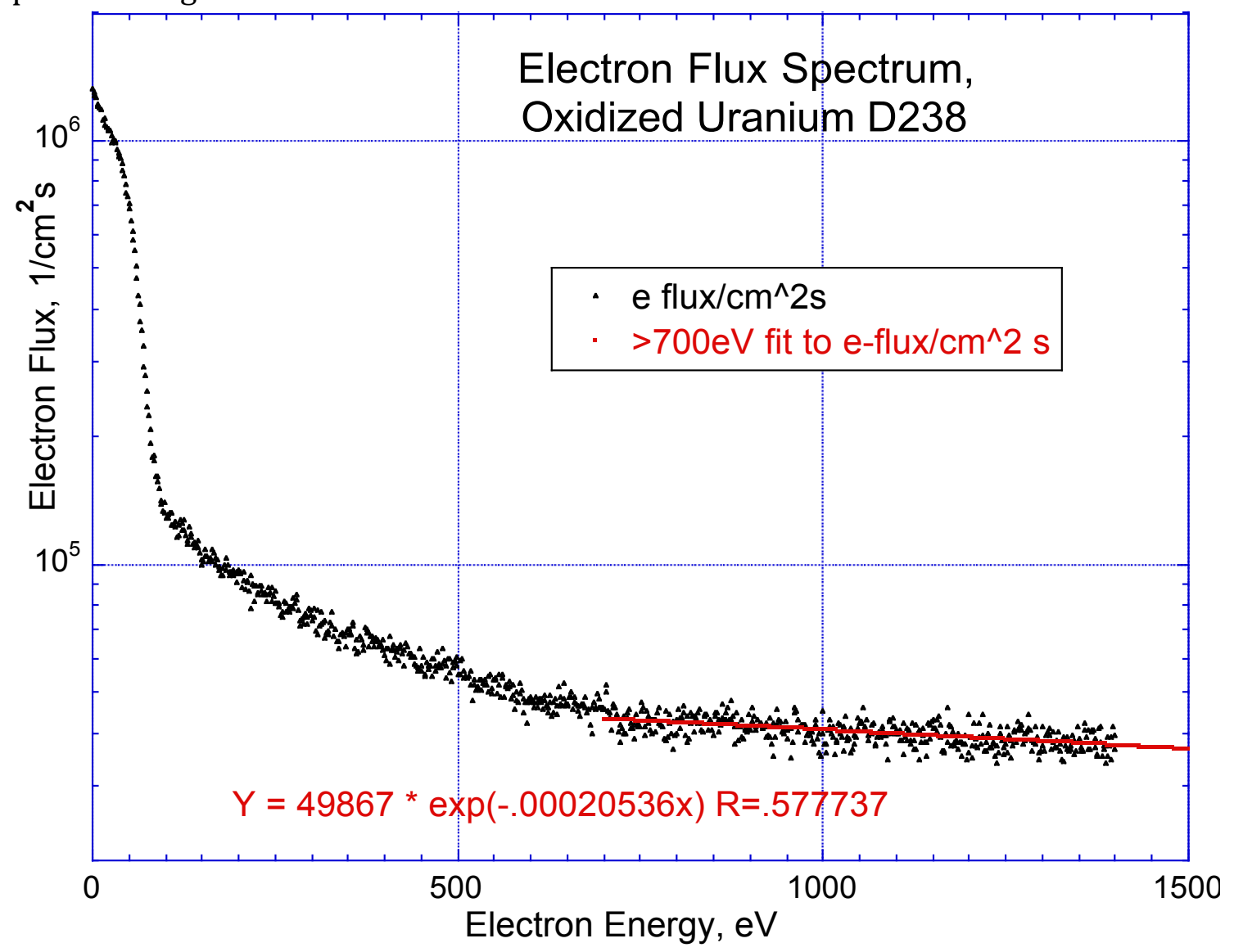

Figure 3. The electron flux $/ \mathrm{cm}^{2}$ s derived from "counts per second" of the spectrometer, together with a fits to the data.

The fit to the data above $700 \mathrm{eV}$ is used to extrapolate to higher electron energies, as shown in figure 4. Having an analytical function describing the energy flux facilitates subsequent calculations that determine radiation induced chemical reaction rates. The total electron current is the integral over the curve in figure $3,2.2^{*} 10^{-11} \mathrm{~A}$. The total energy flux is the integral of the product $\left\{\right.$ e-flux $\left.(E)^{*} \mathrm{E}\right\}$ which yields-> 7.4*10-9

2 J. Moulder, Physical Electronics, USA 
watt $/ \mathrm{cm}^{2}$. Depleted uranium consists mostly of U238 that has a specific activity of $\sim 12400 \mathrm{Becquerel} / \mathrm{g}$ from alpha emission ( $\sim 4.2 \mathrm{MeV}$ U238->Th234), plus 12400 Bequerel/g from beta emission ( $\sim .2 \mathrm{MeV}$ Th234-> Pa 234m) and $\sim 12400$ Bequerel/g from beta emission (2.29MeV Pa234m->U234), a total energy release of $6.69^{*} 12400 \mathrm{MeV} / \mathrm{g} \mathrm{s}=\sim 1.3^{*} 10^{-8} \mathrm{watt} / \mathrm{g}$. Assuming that all of this energy exits from a slice of uranium of $1 \mathrm{~cm}^{2}$ area and $1 \mathrm{~g}$ weight, the maximum energy release per surface is $1.3^{*} 10^{-8} \mathrm{Watt} / \mathrm{cm}^{2}$. The measured energy release from the surface is so close to the total possible release that the measurement and/or the conversion from counts/s to electron flux $/ \mathrm{cm}^{2}$ are suspicious.

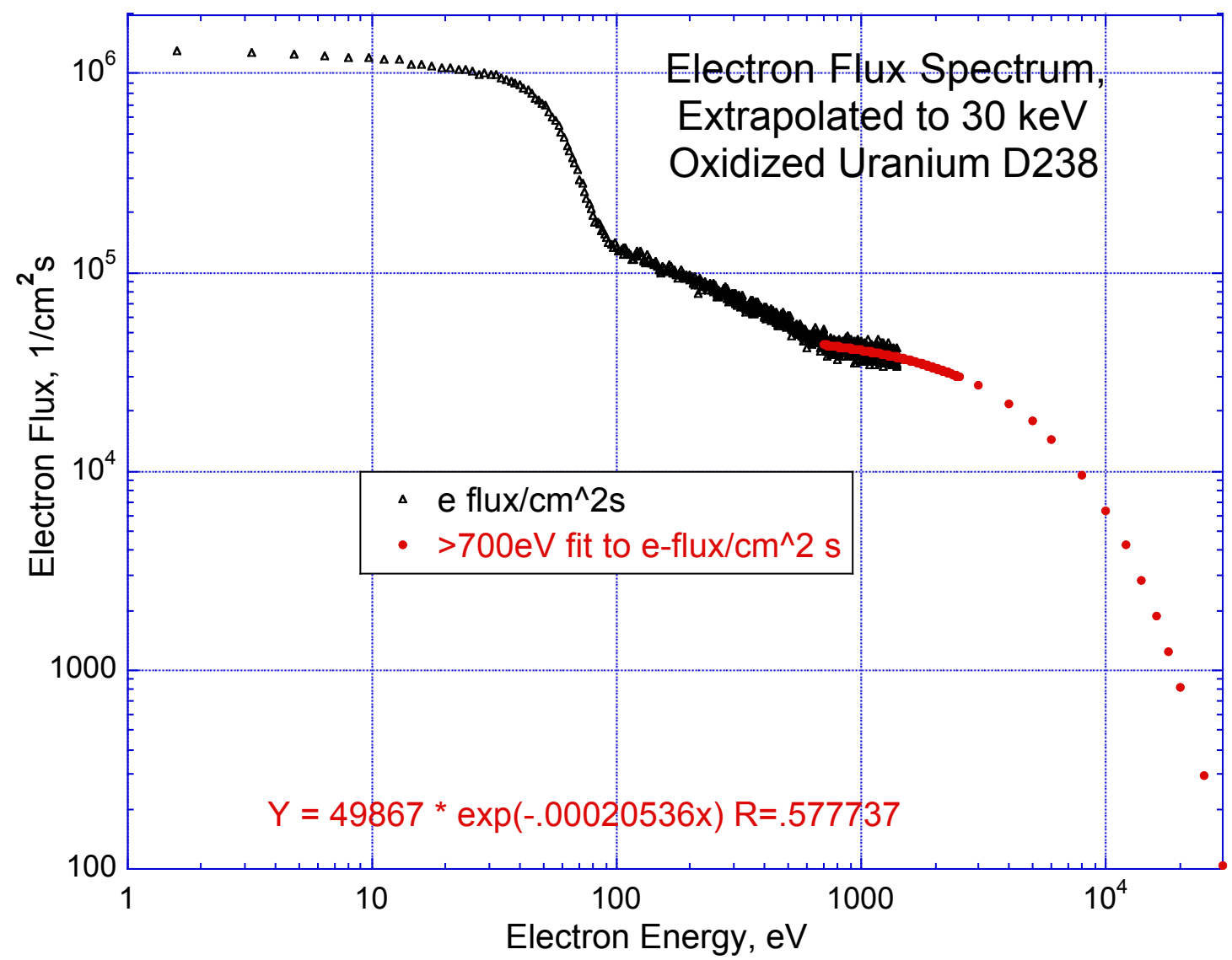

Figure 4. Extrapolation to higher electron energy of the exponential fit to the data between 700 and $1400 \mathrm{eV}$ predicts a very rapid decrease in electron flux with increasing electron energy.

\section{III ) Electron-impact induced reactions in matter (solid, or gas, or adsorbed gas).}

The rate of collisions Rctn(E) (reactions/ $\mathrm{cm}^{2} \mathrm{~s}$ ) caused by electrons of energy $\mathrm{E}$, is determined by equation (1) below: 
$\operatorname{Rctn}(\mathrm{E})\left(1 / \mathrm{cm}^{2} \mathrm{~s}\right)=$ electron flux $(\mathrm{E})\left(\mathrm{e}-/ \mathrm{cm}^{2} \mathrm{~s}\right) * \sigma(E)=$ reaction cross section $\left(\mathrm{cm}^{2}\right)$

$* \mathrm{M}(\mathrm{E})=$ number of accessible reactants $\left(\# / \mathrm{cm}^{2}\right)$

$\mathrm{M}(\mathrm{E})=\left\{\right.$ Number of reactants $\left./ \mathrm{cm}^{3}\right\} *$

"reactive path-length $\mathrm{L}_{e-\text { reactive }}$ " of electrons at energy $\mathrm{E},(\mathrm{cm})$

$* \cos \Theta_{\text {average }}$, the cosine of the angle at which electrons

are emitted from the surface 'on average' ( see figure 1)

The cosine of the average angle of electron emission in equation (1) was calculated in figure 1.

Equation (1) has three factors:

the electron flux,

the reaction cross sections,

and the number of reactancts accessible to an electron of energy $E$.

The flux is described above. The cross sections for hydrogen are available in the literature $\left(\right.$ Yoon $\left.^{3}\right)$. The reactive path length of electrons, Le-reactive, in equation [1] is the amount of matter that electrons travel through with energy sufficient to cause a particular reaction (e. g. to cause dissociation if the matter is gaseous,). This length in equation [1] determines the total number of atoms or molecules that the electrons encounter as they traverse the material, and hence the total number of reaction products they can generate per unit area and unit time. A discussion of the minimum and maximum reaction path length follows. A typical path of a charged particle through matter is shown in figure 5 which illustrates the decrease in energy the charged particle experiences as it travels to its maximum path length, its "range".

3 Yoon, J. S., M. Y. Song, et al. (2008). "Cross sections for electron collisions with hydrogen molecules." Journal of Physical and Chemical Reference Data 37(2): 913931. 


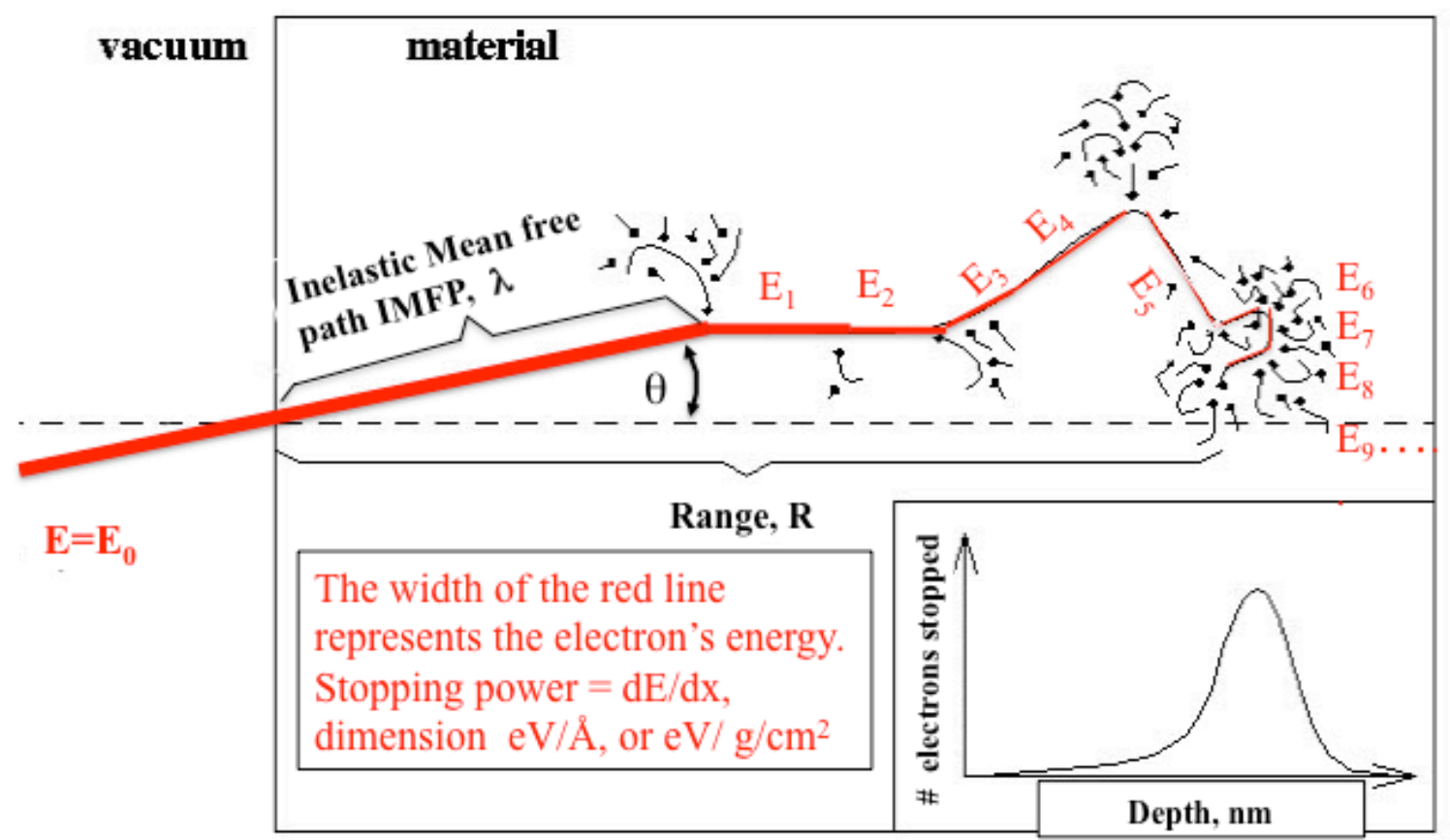

Figure 5. Schematic representation of a high energy positively charged particle's trajectory and associated energy loss along the particle's path, illustrating the concept of "inelastic mean free path 'IMFP'", "range", and "stopping power". The particle's energy is represented by the width of the path, decreasing with each inelastic reactive encounter with matter.

Figure 5 illustrates that a charged particle continues to travel beyond the distance of the first energy-losing encounter, the inelastic mean free path (IMFP), and has typically the majority of its collisions towards the end of its travel, called "range", where its energy is lowest, because at high energy the collision cross sections increase with decreasing energy, see Yoon's graph later in this paper.

A Monte Carlo calculation by Postek ${ }^{4}$ following an electron of $1 \mathrm{keV}$ energy into a silicon substrate is shown in figure 6 depicting the trajectories actually followed, albeit only in two, rather than 3 dimensions. It is impossible to determine from figure 6 what the "IMFP" is, though one can conclude that the "range" is about 30 $\mathrm{nm}$. As will be shown below, calculations predict an IMFP of about $2 \mathrm{~nm}$, and a range of $30 \mathrm{~nm}$. The difference between IMFP and range increases with increasing energy. For example: In hydrogen gas the dissociation energy is $4.5 \mathrm{eV}$, the ionization potential is $15.4 \mathrm{eV}$. Hence an electron with a kinetic energy of $20 \mathrm{eV}$ may loose most of its energy in a single interaction, while an election with keV energy can sustain many such interactions before it is at the end of its range.

${ }^{4}$ Postek, M. T. and A. E. Vladar (2011). "Modeling for Accurate Dimensional Scanning Electron Microscope Metrology: Then and Now." Scanning 33(3): 111-125. 


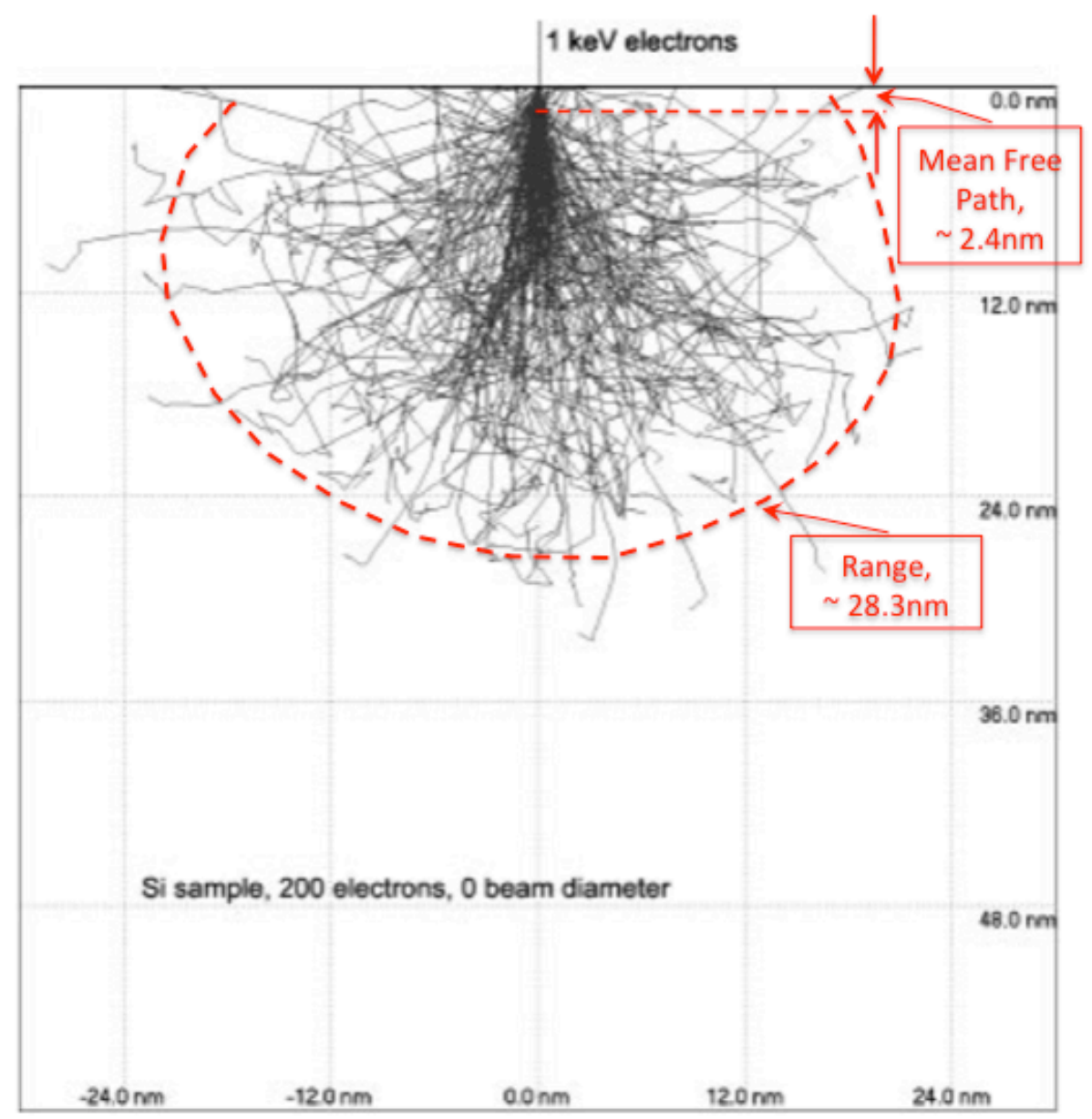

Figure 6. Schematic representation of the trajectory of an electron with $1 \mathrm{keV}$ energy traversing silicon. After Postek ${ }^{5}$.

At minimum Le-reactive is the "inelastic electron mean free path [IMFP]". This "IMFP" is the path-length, expressed either as matter mass traversed (e.g. $\left[\mathrm{g} / \mathrm{cm}^{2}\right]$, number of reactants passed by [\#reactants $/ \mathrm{cm}^{2}$ ], or simply - for a material of known density - length [cm,nm, $\mathrm{A}]$ ) at which "in the mean" electrons experience the first energy-losing interaction, discussed in III. 1) and III.2) below. The energy loss may be small or large. At maximum, Le-reactive is the mass "range" at which electrons are reduced to thermal energy, discussed in section $\mathrm{V}$ ) below. It is apparent from both figure 5 and 6 that there can be a large difference between IMFP and range.

IV) The minimum Le-reactive is the "inelastic electron mean free path, 'IMFP'”, $\lambda\left(\mathrm{g} / \mathrm{cm}^{2}, \# / \mathrm{cm}^{2}\right.$, number of monolayers, number of accessible molecules or atoms, or $\AA$ )

\section{1) The mean free path derived from total inelastic cross section data.}

5 Ibid. 
If all cross sections for inelastic electron-matter collisions are known, the inelastic mean free path (in "units" of 'number of accessible molecules or atoms') can be derived from equation (1). :

$\operatorname{Rctn}(\mathrm{E})=$ the number of reactions $/ \mathrm{cm}^{2} \mathrm{~s}$ is given by

$$
\begin{aligned}
\operatorname{Rctn}(\mathrm{E})\left(1 / \mathrm{cm}^{2} \mathrm{~s}\right) & =\text { electron flux }(\mathrm{E})\left(1 / \mathrm{cm}^{2} \mathrm{~s}\right) * \sigma_{\text {TOTAL INELASTIC }}(\mathrm{E})\left(\mathrm{cm}^{2}\right) * \mathrm{M}(\mathrm{E})\left(1 / \mathrm{cm}^{2}\right) \\
\mathrm{M}(\mathrm{E}) & =\text { number of accessible reactants } / \mathrm{cm}^{2} \\
& =\text { density }\left(\mathrm{g} / \mathrm{cm}^{3}\right) *(\text { Avogadro's number } / \text { Molecular weigth }(\mathrm{g})) * \operatorname{IMFP}(\mathrm{cm})
\end{aligned}
$$

By definition the inelastic mean free path IMFP has been reached when one half of the electron flux has experienced an energy-losing 'inelastic' collision, i.e. when $\operatorname{Rctn}(E)\left(1 / \mathrm{cm}^{2} \mathrm{~s}\right)=(1 / 2)$ electron flux $(\mathrm{E})\left(1 / \mathrm{cm}^{2} \mathrm{~s}\right)$. Therefore when:

$$
\begin{aligned}
\frac{1}{2} \text { electron flux }(\mathrm{E}) & =\text { electron flux }(\mathrm{E}) * \sigma_{\text {TOTAL INELASTIC }} * \mathrm{M}(\mathrm{E}) . \text { Hence } \\
\mathrm{M}(\mathrm{E}) & =1 /\left(2 * \sigma_{\text {TOTAL INELASTIC }}\right)=\lambda\left(\text { reactants } / \mathrm{cm}^{2}\right)
\end{aligned}
$$

Equation [2] is only valid if only inelastic cross sections are included in the total cross section.

\section{2) The inelastic mean free path derived from optical data.}

The inelastic mean free path of electrons is of interest in all electron spectroscopy techniques \{e.g. Auger Spectroscopy (AES), Photo Electron Spectroscopy (XPS)\} since it determines the thickness of the surface layer sampled by the technique. Many sophisticated calculations of the IMFP for materials based on optical data have been done since Seah and Dench ${ }^{6}$ derived IMFP's by fitting curves to experimental data.\{see Powell et al. ${ }^{7}$ (2011) for IMFP calculations of 41 elements and references therein\}. Figure 7 shows Powell's IMFP curves (in units of $\AA$ A) for 41 elements. (from Lithium to Bismuth). Alkali metals produce curves that deviate most from the "typical" pattern. Despite these differences, the IMFP's do NOT differ dramatically, as figure 7 below shows, keeping in mind, however, that figure 7 is a double logarithmic plot. The difference between elements in figure 7 would be much less if the IMFP were expressed in $\mathrm{g} / \mathrm{cm}^{2}$ instead of in $\AA$.

\footnotetext{
${ }^{6}$ Seah, M. P. Dench W. (1979). "Quantitative electron spectroscopy of surfaces: a standard data base for electron inelastic mean free paths in solids" Surface and interface analysis [0142-2421] vol.1, (no.1 ): pg:2 -11. ${ }^{7}$ Powell, C. J., S. Tanuma, et al. (2011). "Calculations of electron inelastic mean free paths. IX. Data for 41 elemental solids over the $50 \mathrm{eV}$ to $30 \mathrm{keV}$ range." Surface and Interface Analysis 43(3): 689-713.
} 


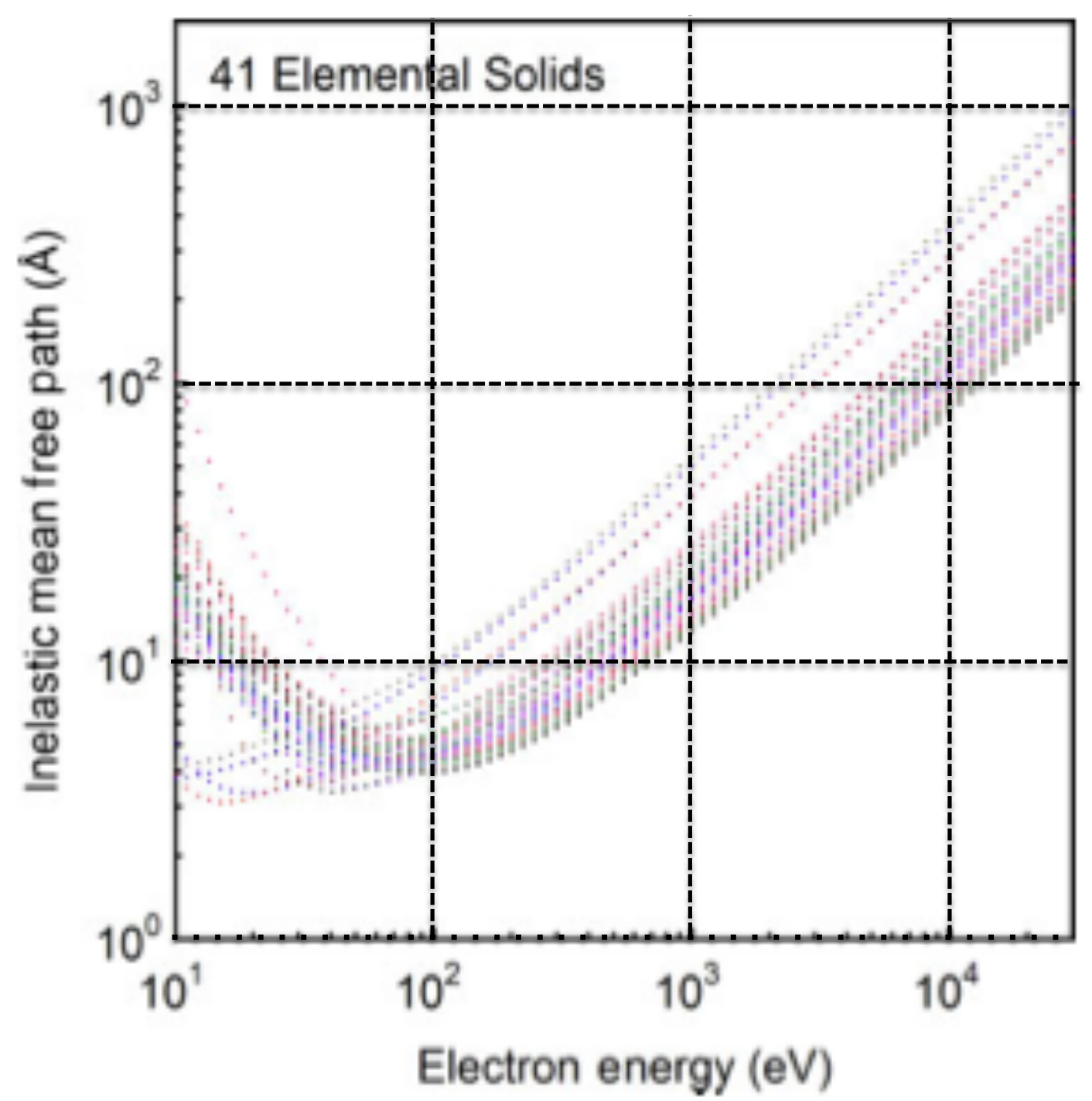

Figure 7. IMFP for 41 elements, after Powell et al.

\section{V) The mean free path of molecules in the gas phase.}

It is sometimes of interest to determine the number of reaction products within the reactant's mean free path, rather than the number of reactants in the electron's mean free path, since one half of the reaction products within the reaction products mean free path will strike the substrate without any collision with other molecules, and potentially initiate a reaction on the surface. . The mean free path of molecules in a gas $\lambda_{\text {molecule }}=1 /\left(2{ }^{5} \pi^{*} \mathrm{~d}_{0}{ }^{2} \mathrm{n}\right)$, where $\mathrm{n}$ is the number density and $\mathrm{d}_{0}$ is the molecular diameter. \{e.g. $\mathrm{d}_{0}=\left(\sim 2 \rightarrow 6^{*} 10^{-8} \mathrm{~cm}, \sim 2.93^{*} 10^{-8} \mathrm{~cm}\right.$ for hydrogen. The diameter of the hydrogen atom is about twice the Bohr diameter, i.e $1.1 \AA$ \}. Hence the number of hydrogen molecules within a distance $\lambda_{\text {molecule }}$ from a surface of area $1 \mathrm{~cm}^{2}$ is $=\lambda_{\text {molecule }}{ }^{*} \mathrm{n}=1 /\left(2.5 \mathrm{\pi}^{*} \mathrm{~d}_{0}{ }^{2}\right)=5.63 * 10^{14} / \mathrm{cm}^{2}$ for hydrogen, $=\sim<$ the 
equivalent of one monolayer adsorbed onto a surface. This number is independent of pressure. Only one half of the reaction products travel in a direction towards the surface, assuming that the reaction with electrons that produces the reaction producet did NOT generate a preferred direction for the reaction products.

\section{VI) The maximum Le-reactive, the mass range at which electrons are reduced to thermal energy, the "range".}

As stated above, the inelastic mean free path range defines the path length of electrons, Le-reactive, over which on average half the electrons experience an energylosing interaction with matter. The maximum path length is given by the electron "range", the path length at which electrons are reduced to thermal energy (see figure 5) .

\section{VI.1) The range of electrons in hydrogen, NIST data.}

Figure 8 shows the range of electrons and the stopping power for electrons in hydrogen (NIST data).

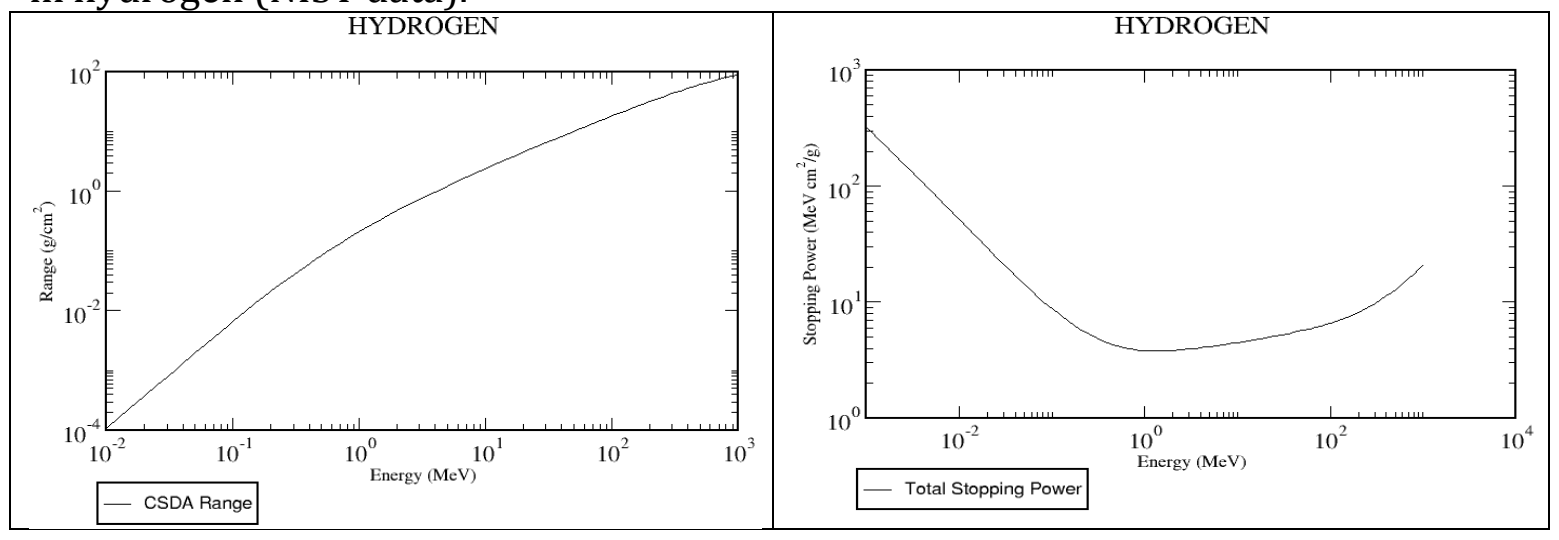

Figure 8. Left image: "CSDA =Continuous Slowing Down" range $\mathrm{g} / \mathrm{cm}^{\wedge} 2$ of electrons in hydrogen, with electron energies from .01 to $1000 \mathrm{MeV}$. Right image: "Total Stopping Power" , $\mathrm{MeV} \mathrm{cm}^{\wedge} 2 / \mathrm{g}$, for hydrogen. The NIST site provides stopping power for energies higher than $1 \mathrm{keV}$, but range only for energies above $10 \mathrm{keV}$. From www.nist.gov/PhysRefData/Star/Text/ESTAR.html

As is discussed in detail at NIST's website, range calculations become increasingly more unreliable with decreasing energy "due to the lack of shell corrections which are required when the velocity of the incident electron is no longer large compared to the velocities of the atomic electrons, especially those in the inner shells." Therefore NIST stops the CSDA curves at $10 \mathrm{keV}$. Nevertheless, the same NIST site shows stopping power curves down to $1 \mathrm{keV}$, see figure 8 .

\section{VI.2) Comparison of $1 \mathrm{keV}$ electron range in silicon (NIST data) with Monte Carlo Calculations, and with the calculated inelastic mean free path.}

To make a visual comparison of the calculated IMFP's of Powell, and NIST's range, we extend NIST's CSDA range curve for silicon to energies below $10 \mathrm{keV}$ and 
plot the IMFP derived from Powell et al.'s data and CSDA range from NIST for silicon in figure 9. We use silicon for comparison since a Monte Carlo calculation for silicon is available at $1 \mathrm{keV}$ (see figure 6). Comparison between the IMFP and range at 1 $\mathrm{keV}$ in figure 9 provides a quantitative measure of the difference between mean free path and range, complementing the graphic depiction in figure 5. In addition, the agreement between the range observed for silicon in figure 6 and the range in figure 9 derived from NIST's data suggests that at least down to $1 \mathrm{keV}$ the extrapolation of NIST's range data is reasonable.

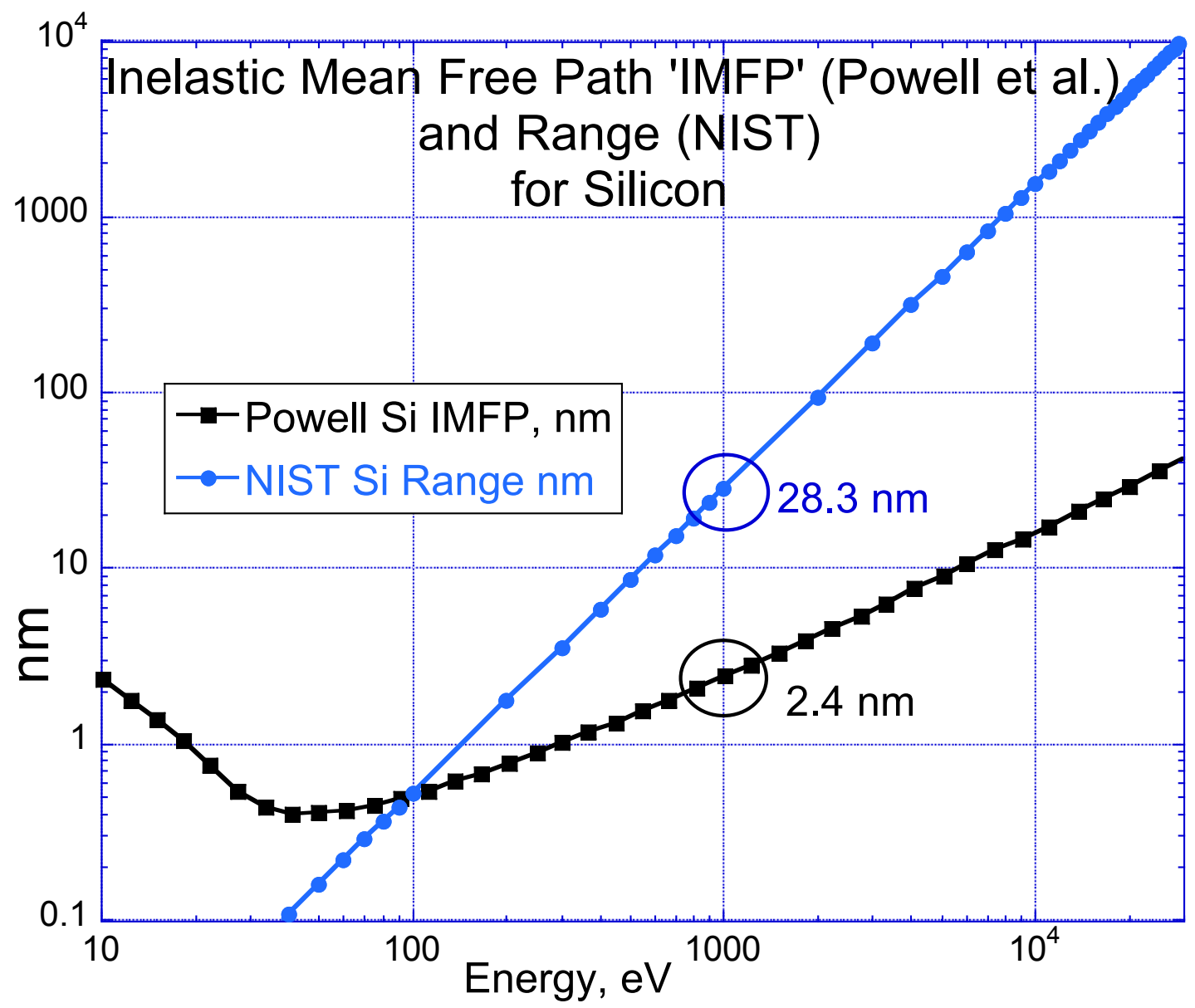

Figure 9. Comparison of the inelastic mean free path and the range of electrons in silicon.

Clearly it is NOT reasonable to extrapolate the range to energies to $100 \mathrm{eV}$, since the range must be at least twice the mean free path because at the mean free path only half the electrons have undergone an "energy-losing" collision. The "energy-losing" collision does NOT in many icases reduce the electron's energy to thermal energy. For instance, an electron impact ionization of hydrogen requires $\sim 15 \mathrm{eV}$, hence a $100 \mathrm{eV}$ electron can induce at least 6 six ionizations before it is reduced to thermal energy. Since both NIST's data and Powell's calculations are consistent with experimental data and with Monte Carlo calculations for silicon, one can conclude from figure 9 that at $1 \mathrm{keV}$ the an electron has more than 12 (28.3 nm divided by 
$2.4 \mathrm{~nm}$ ) collisions before it is thermalized. Many more than 12 , because with each collision its energy decreases by $\Delta \mathrm{E}$, and its inelastic mean free path at $\mathrm{E}-\Delta \mathrm{E}$ is shorter than at $E=1 \mathrm{keV}$, until its energy has decreased to below $100 \mathrm{eV}$, where Powell's IMFP has a minimum.

\section{VII) Reaction of electrons emanating from a uranium surface with hydrogen molecules.}

The cross sections for e- $\rightarrow \mathrm{H}_{2}$ reactions are given in Yoon ${ }^{8}$ and shown in graphical from in figure 10. In figure 10 the total cross section, labeled "tot" includes both elastic (lableled "elas") and inelastic collisions (all other collisions). The energy loss in momentum transfer collisions (labeled "mom transf") is very small, since the mass of the electron is about two thousand times smaller than the mass of a hydrogen molecule and will NOT be included as an inelastic collision.

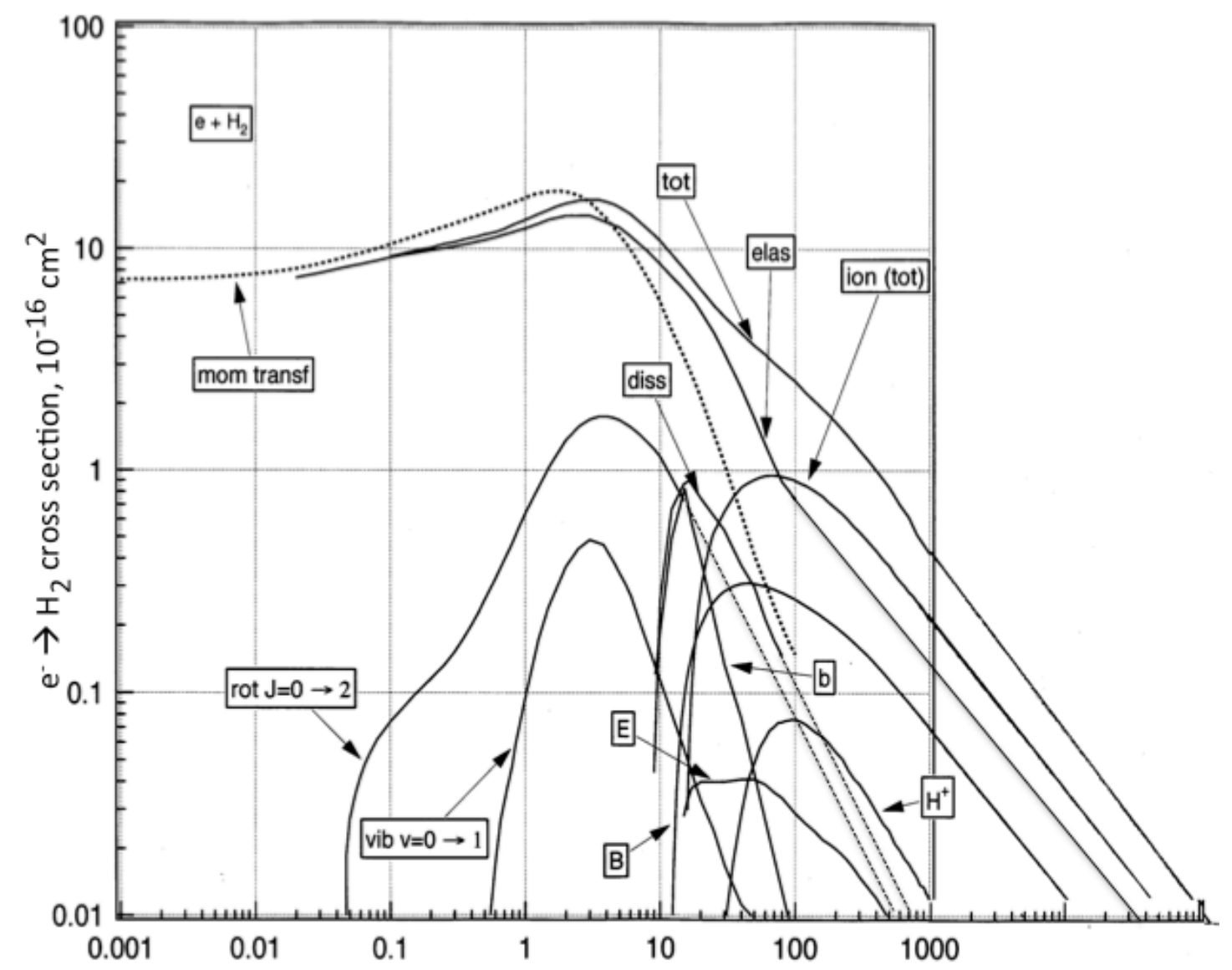

Figure 10. Reaction cross sections of electrons with hydrogen molecules as a function of electron energy. Some of the cross-sections are extended beyond their original range or beyond their maximum energy ( $1 \mathrm{keV})$ of the original graph using a continuation of their slope within the graph.

8 ibid. 
Figure 10 indicates that reactions of practical interest, e.g. ionization, dissociation, have significant cross sections only above about $9 \mathrm{eV}$ electron energy.

In section III.1) it was pointed out that only the total inelastic cross section is relevant for determining the inelastic mean free path.

Figure 11 shows the total inelastic cross section for hydrogen, derived by subtracting the elastic cross section in figure 10 from the total cross section.

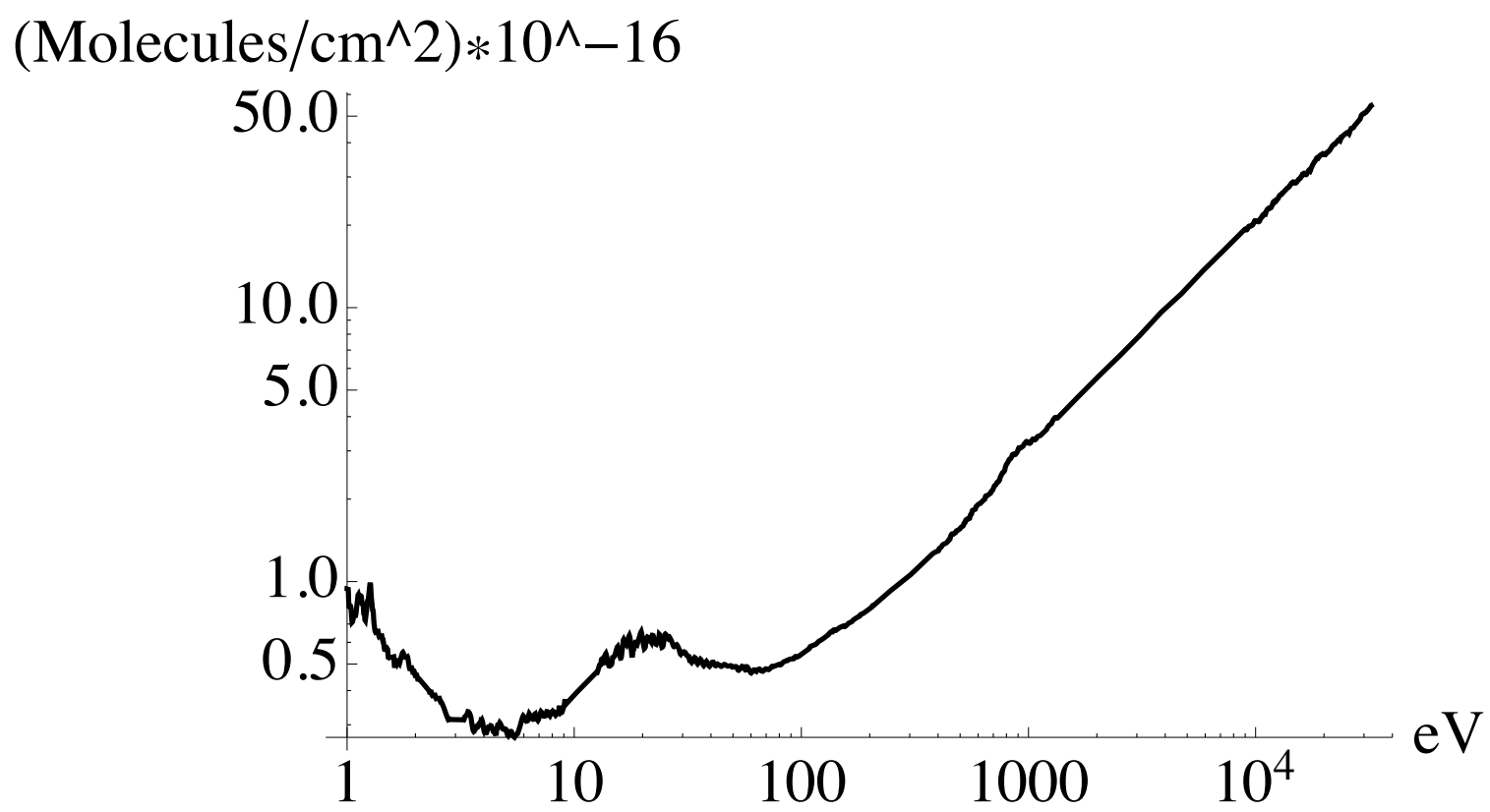

Figure 11. The total inelastic cross section of hydrogen, expressed as molecules $/ \mathrm{cm}^{2}$ After Yoon et al.

\section{VII.1) The "minimum number of reactions", i. e the reactions that occur within the inelastic mean free path.}

In figure 12 the inelastic mean free path of electrons in hydrogen is plotted using the total inelastic cross section shown in figure 11. Also shown is the electron range in hydrogen derived from NIST data by converting NIST's range given in $\mathrm{g} / \mathrm{cm}^{2}$ to 'number of hydrogen molecules/ $\mathrm{cm}^{2}$ '. Furthermore Powell's IMFP data for Lithium are plotted also for comparison after converting $\AA$, the IMFP unit used by Powell, to 'number of hydrogen molecules $/ \mathrm{cm}^{2}$ ' by assuming solid hydrogen at $.088 \mathrm{~g} / \mathrm{cm}^{3}$. Lithium is used for comparison, since all elements in the first column of the periodic table (the Alkali metals) show very similar behavior, and since Lithium is closest in density to solid hydrogen. 


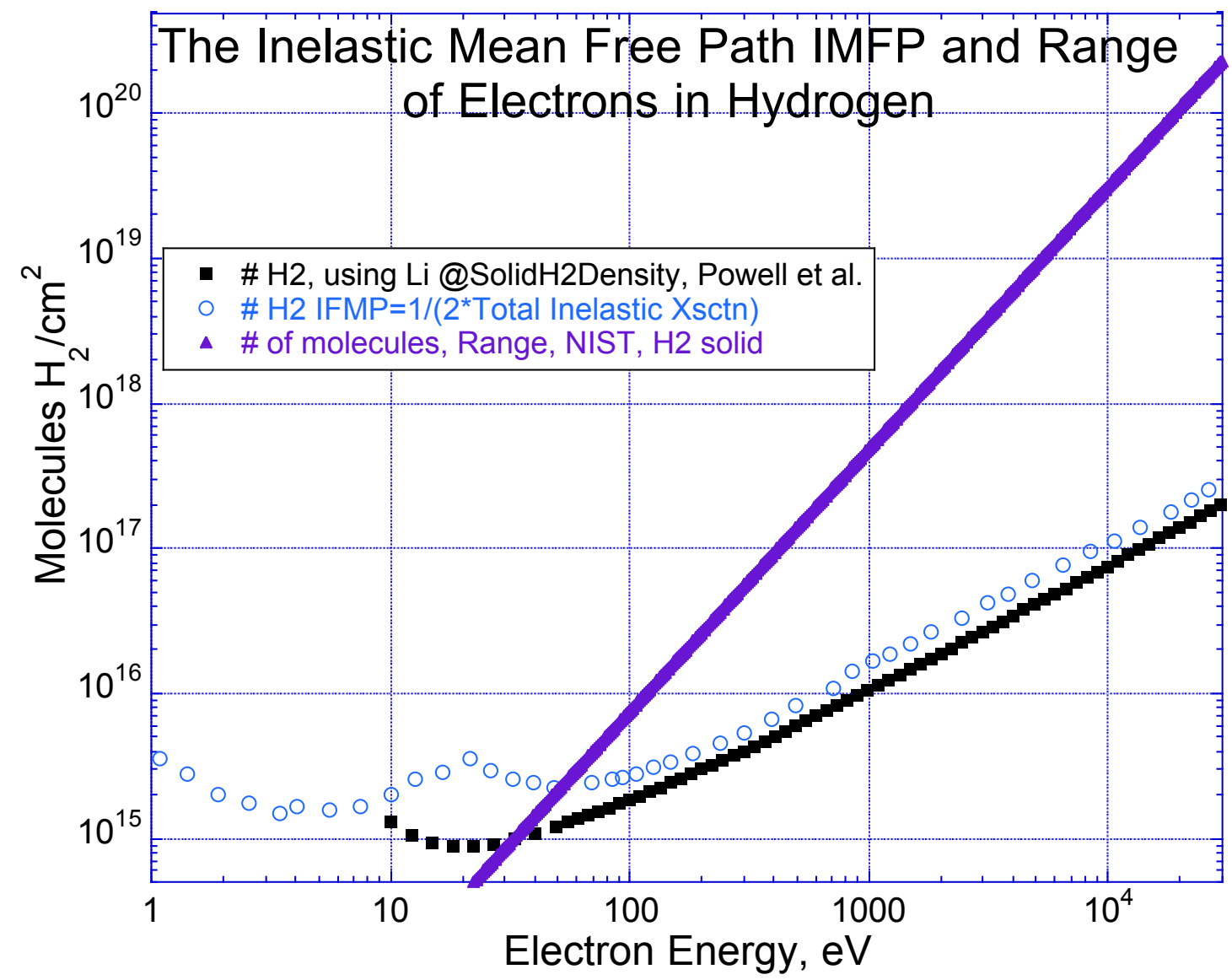

Figure 12. Comparison of the inelastic mean free path calculated from the total inelastic cross section derived from Yoon et al., and of that derived from Powell's calculation for Lithium (at solid hydrogen density) with NIST's range.

Figure 12 demonstrates that the concept of deriving the IMFP from measured total inelastic cross sections is consistent in magnitude and energy dependence with the IMFP derived from Powell's calculations. The NIST range is included to illustrate that electrons will undergo many collisions - and create many more reaction products than occur in the IMFP - before they are thermalized.

The number of reaction products Rctn(E) occuring within the energy dependent inelastic mean free path are now calculated at each energy E, using equation (1), employing the energy dependent electron flux, electron mean free path, and Yoon's cross section $\sigma(\mathrm{E})$ of each type of reaction. The total number "Rctn", e.g. for the dissociation reaction, is found by integrating Rctn dissociation$_{\text {(E) over }}$ the energy range where dissociation cross sections exist, i.e. Rctn dissociation $\int$ Rctn $_{\text {dissociation }}(\mathrm{E}) \mathrm{dE}$. The "Rctn" values for a few electron induced reaction products are listed in Table 1 below. The program "Mathematica" is used for the numerical integration. Mathematica interpolates between energy values for each set of data, so that data sets with different energy-value lists can be used. 


\begin{tabular}{|c|c|c|c|}
\hline $\begin{array}{l}\text { Cell Type: } \\
\text { U surface facing } \mathrm{H}_{2} \text { gas }\end{array}$ & $\begin{array}{l}\text { Rctn }_{\text {All Ionization }} \\
\mathrm{H}_{2}+\mathrm{e}_{-} \rightarrow \\
\mathrm{H}_{2}+\mathrm{H}^{+}\end{array}$ & $\begin{array}{l}\text { Rctn }_{\mathrm{H} 2-\rightarrow \mathrm{H}+\text { only }} \\
\mathrm{H}_{2}+\mathrm{e}^{-} \rightarrow \mathrm{H}^{+}\end{array}$ & $\begin{array}{l}\mathrm{R}_{\text {Dissociation }} \\
\mathrm{H}_{2}+\mathrm{e}-\mathrm{2H}\end{array}$ \\
\hline $\begin{array}{l}\mathrm{Rctn} / \mathrm{cm}^{2} \mathrm{~s}=\# \text { of } \mathrm{H}_{2} \\
\text { reacted within electron } \\
\text { inelastic mean free path } \\
=1 / 2^{*} \text { (Total Inelastic } \\
\text { Cross Section) }\end{array}$ & $2.1 * e+8$ & $4.3^{*} 10^{\wedge} 6$ & $1.6^{*} 10^{\wedge} 7$ \\
\hline $\begin{array}{l}\text { Rctn } / \mathrm{cm}^{2} \mathrm{~s}=\# \text { of } \mathrm{H}_{2} \\
\text { reacted within electron } \\
\text { inelastic mean free path } \\
\text { derived from Powell et } \\
\text { al. }\end{array}$ & $7.2 * 10 \wedge 7$ & $1.5 * 10 \wedge 6$ & $3.3^{*} 10^{\wedge} 6$ \\
\hline $\begin{array}{l}\mathrm{Rctn} / \mathrm{cm}^{2} \mathrm{~s}=\# \text { of } \mathrm{H}_{2} \\
\text { reacted within mean free } \\
\text { path of hydrogen } \\
\text { molecules in hydrogen } \\
\text { gas }\end{array}$ & $4.4 * 10 \wedge 6$ & $2.0 * 10 \wedge 5$ & $1.5^{*} 10^{\wedge} 6$ \\
\hline $\begin{array}{l}\mathrm{Rctn} / \mathrm{cm}^{2} \mathrm{~s}=\# \text { of } \mathrm{H}_{2} \\
\text { reacted within electron } \\
\text { inelastic mean free path } \\
=1 / 2^{*}(\text { Total Inelastic } \\
\text { Cross Section) } \\
\text { for } 7-100 \mathrm{eV} \text {, plus within } \\
\text { the range for } 100-> \\
3000 \mathrm{eV}\end{array}$ & $1.5^{*} 10 \wedge 10$ & $1.5 * 10 \wedge 10$ & $2.0^{*} 10^{\wedge} 7$ \\
\hline
\end{tabular}

Table 1. The "Rctn" = reaction values for ionization and dissociation between $\mathrm{H}_{2}$ and electrons for various inelastic path models.

Table 1 lists in the first 3 rows the reactions within the mean free path IMFP. By definition of the IMFP, only one half of the electrons have encountered their first reaction.

The first row uses the IMFP based on the total inelastic cross section derived from Yoon's data, the second row uses Powell et al.'s IMFP. The third row uses the mean free path of hydrogen molecules in hydrogen gas, to make an estimate of how many reaction products may reach the surface within undergoing a collision.

The last row in table 1 makes a guess at the reactions within the range rathern than the IMFP of electrons, (see figure 12) by calculating reactions with electrons with energies between 7 and $100 \mathrm{eV}$ based on the mean free path of row 1 , and reactions between $100 \mathrm{eV}$ and $30 \mathrm{keV}$ based on the NIST range. The "guess" is an underestimate, because the electron looses energy as it travels through the range, and the cross section for reaction increases with decreasing energy. 


\section{VII.2) The "maximum number of reactions", i. e the reactions that occur within the "total energy range" (NOT "range=molecules $/ \mathrm{cm}^{2} \mathrm{~s}$ ") of electrons.}

A more realistic estimate of the reactions occurring within the energy range of electrons incorporates the change in mean electron energy and the associated change in reaction cross section and in the inelastic mean free path (expressed in molecules $/ \mathrm{cm}^{2}$ ) occurring with each energy-losing inelastic collision. Assume that the change in energy in each inelastic collision is $\Delta \mathrm{E}$. The number of collisions " $\mathrm{n}$ " that can occur for an electron of energy $E$ is then $n=E / \Delta E$, where $n$ is the integer part of the fraction, and $n=0$ for $E / \Delta E<1$. To visualize the process, see figure 12: An electron entering the gas with $1 \mathrm{keV}$ energy has its first collision while traversing the IMFP(E) number of molecules shown in figure 11 and 12 at $1 \mathrm{keV}$ with the reaction probability defined by Yoon's crossection at $1 \mathrm{keV}$ (see figure 10), looses on average $\Delta \mathrm{E} \mathrm{eV}$, continues to traverse the next IMFP at (1keV- $\Delta \mathrm{E})$, reacts within the IMFP( $1 \mathrm{keV}-\Delta \mathrm{E})$ with the probability defined by the cross section at (1keV- $\Delta \mathrm{E})$, looses on average $\Delta \mathrm{E}$, continues with energy (1keV-2 $\Delta \mathrm{E})$... etc., until it has reached an energy so low that further reactions of interest are impossible. With that assumption, the number of reactions due to electrons of any energy $E$ is given by equation [3] below:

$\Delta \mathrm{E}=$ mean energy loss per inelastic collision

IMFP = inelastic mean free path

$|n(E)|=|E / \Delta E|$, where $|n(E)|$ is an integer with minimum value $=0$

$\operatorname{Rctn}(E)=\operatorname{Eflux}(E)^{*} \int_{E \min }^{E \max } \sum_{n=0}^{n=|n(E)|} \sigma\left(E-n^{*} \Delta E\right) * M\left(E-n^{*} \Delta E\right) d E$

$\operatorname{Rctn}(E)=$ number of reactions $/ \mathrm{cm}^{2} \mathrm{~s}$

$\operatorname{Eflux}(E)=$ electron flux at energy $\mathrm{E}, 1 / \mathrm{cm}^{2}$

$\sigma(E)=$ cross section of the reaction of interest, $\mathrm{cm}^{2}$

$M(E)=$ number of accessible molecules per IMFP, $1 / \mathrm{cm}^{2}$

$E \min =$ minimum energy of electron flux

$E \max =$ maximum energy of electron flux 


\begin{tabular}{|l|l|l|l|}
\hline $\begin{array}{l}\text { Cell Type: } \\
\text { U surface facing } \mathbf{H}_{2} \text { gas }\end{array}$ & $\begin{array}{l}\text { Rctn } \\
\mathbf{H}_{2}+\mathbf{e}-\rightarrow \\
\mathbf{H}_{2}{ }^{+} \text {\& } \mathbf{H}^{+}\end{array}$ & $\begin{array}{l}\text { Rctnization } \\
\mathbf{H}_{2}+\mathbf{e}-\rightarrow \mathbf{H}^{+} \text {only }\end{array}$ & $\begin{array}{l}\mathbf{R}_{\text {Dissociation }} \\
\mathbf{H}_{2}+\mathbf{e}-\rightarrow 2 \mathbf{H}\end{array}$ \\
\hline $\begin{array}{l}\text { Rctn/cm }{ }^{2} \mathrm{~s}=\# \text { of } \mathrm{H}_{2} \\
\text { reacted within NIST } \\
\text { electron ENERGY range } \\
\text { using inelastic mean free } \\
\text { path derived from Powell } \\
\text { et al. }\end{array}$ & $1.3^{*} 10 \wedge 10$ & $2.8^{*} 10 \wedge 8$ & $1.8^{*} 10^{\wedge} 9$ \\
\hline
\end{tabular}

Table 2. The "maximum" number of reactions $/ \mathrm{cm}^{2} \mathrm{~s}$ based on the assumption that electrons loose an energy of $\Delta \mathrm{E}=20 \mathrm{eV}$ in each mean free path and continue to react at successively lower energy until they are below the energy where inelastic collisions can occur.

The program "Mathematica" is used to solve equation [3] for the numerical integration and summation, using a working precision of 60 digits.

The reaction rates "Rctn" are nevertheless only estimates, since the energy loss $\Delta \mathrm{E}=20 \mathrm{eV}$ in each mean free path is only a guess. Taking $\Delta \mathrm{E}=16 \mathrm{eV}$, closer to the ionization energy of $\mathrm{H}_{2}$, increases the rate for ionization to $\mathrm{H}^{+}$to $3.5^{*} 10^{8}$ from $2.8^{*} 10^{8} / \mathrm{cm}^{2} \mathrm{~s}$.

\section{Summary}

The spectroscopically measured emission of electrons from depleted uranium generated by its natural radioactivity is surprisingly high. The electron flux derived from the spectroscopy data together with known reaction cross sections for hydrogen leads to substantial dissociation and ionization reactions per $\mathrm{cm}^{2}$ in the mean free path of those electrons.

However the electron energy flux, $\int$ flux(E)*E*dE integrated from 0 to $1400 \mathrm{eV}$, is close to the total energy generated by natural radioactivity of one gram of U238. Therefore the results must be used with caution until they are confirmed by other measurements, such as, e.g. measurement of the angular distribution of electron emission and of the total electrical current emitted by the sample.

A likely source of error is the assumption that the electron emission has a cosine angular distribution. 